\title{
The influence of the degree of heterogeneity on the elastic properties of random sphere packings
}

\author{
Journal Article \\ Author(s): \\ Schenker, Iwan; Filser, Frank T.; Hütter, Markus; Gauckler, Ludwig J. \\ Publication date: \\ 2012-05 \\ Permanent link: \\ https://doi.org/10.3929/ethz-b-000048820 \\ Rights / license: \\ In Copyright - Non-Commercial Use Permitted \\ Originally published in: \\ Granular Matter 14(3), https://doi.org/10.1007/s10035-012-0316-5
}




\title{
The influence of the degree of heterogeneity on the elastic properties of random sphere packings
}

\author{
Iwan Schenker • Frank T. Filser • Markus Hütter • \\ Ludwig J. Gauckler
}

Received: 11 December 2010 / Published online: 11 February 2012

(C) Springer-Verlag 2012

\begin{abstract}
The macroscopic mechanical properties of colloidal particle gels strongly depend on the local arrangement of the powder particles. Experiments have shown that more heterogeneous microstructures exhibit up to one order of magnitude higher elastic properties than their more homogeneous counterparts at equal volume fraction. In this paper, packings of spherical particles are used as model structures to computationally investigate the elastic properties of coagulated particle gels as a function of their degree of heterogeneity. The discrete element model comprises a linear elastic contact law, particle bonding and damping. The simulation parameters were calibrated using a homogeneous and a heterogeneous microstructure originating from earlier Brownian dynamics simulations. A systematic study of the elastic properties as a function of the degree of heterogeneity was performed using two sets of microstructures obtained from Brownian dynamics simulation and from the void expansion method. Both sets cover a broad and to a large extent overlapping range of degrees of heterogeneity. The simulations have shown that the elastic properties as a function of the degree of heterogeneity are independent of the structure generation algorithm and that the relation between the shear modulus and the degree of heterogeneity can be well described by a power law. This suggests the presence of a critical degree of heter-
\end{abstract}

Electronic supplementary material The online version of this article (doi:10.1007/s10035-012-0316-5) contains supplementary material, which is available to authorized users.

I. Schenker $(\varangle) \cdot$ F. T. Filser · L. J. Gauckler

Department of Materials, Nonmetallic Materials,

ETH Zurich, 8093 Zurich, Switzerland

e-mail: iwan.schenker@alumni.ethz.ch

M. Hütter

Materials Technology, Mechanical Engineering, Eindhoven University of Technology, 5600 MB Eindhoven, The Netherlands ogeneity and, therefore, a phase transition between a phase with finite and one with zero elastic properties.

Keywords Degree of heterogeneity .

Mechanical properties · Discrete element method .

Granular material · Porosity

\section{Introduction}

Random sphere packings are ubiquitous model systems for the study of the structural and mechanical properties of granular matter or colloids. Geomechanics [1], granular flow [2] and mixing and segregation of granular materials [3] are but a few examples. The mechanical properties of granular systems depend on various parameters such as the volume fraction [4], the particle size distribution [5], material properties as, for example, the particles' friction coefficients [6] or adhesive forces [7]. Furthermore, as predicted in [8] and discussed in more detail in the following, the mechanical properties strongly depend on the microstructure, i.e., the local arrangement of the particles.

The influence of the microstructure on the mechanical properties is often observed implicitly in experimental and computational mechanical tests on structures differing in preparation history. Macroscopic stress profiles, for example, where found to strongly depend on the sample preparation procedure and thus its microstructure [9]. However, systematic investigations of the mechanical properties as a function of the microstructure are scarce, for two reasons: first, a systematic study of the microstructure-dependent mechanical properties requires the possibility of an unambiguous characterization of the microstructural arrangement of the particles. Second, and equally important, it relies on the possibility of a reproducible generation of microstructures with distinct local arrangements of the particles at constant volume fraction. 
In [10], the concept of the degree of heterogeneity (DOH) has been introduced, constituting a scalar measure of the heterogeneity of the microstructural arrangement of a sphere packing. Three distinct structure characterization methods in conjunction with parameters in fit functions or integrals were shown to allow for a clear quantification and thus classification of the DOH of particle structures. In contrast to distributional structure characterization methods, such as the pair correlation function [11], the bond angle distribution [11], the common neighbors distribution [12] or the Minkowski functionals [13], the concept of the DOH does not rely on the interpretation of distribution curves. This quantifiability is seen as an advantage in view of a correlation of the mechanical to the structural properties.

Experimentally, the reproducible control of the DOH of colloidal microstructures with volume fractions between 0.2 and 0.6 is achieved using direct coagulation casting $[14,15]$, which is an in situ enzyme-catalyzed destabilization method. It allows for the coagulation of electrostatically stabilized colloidal suspensions to stiff particle structures by either shifting the $\mathrm{pH}$ of the suspension to the particles' isoelectric point or by increasing the ionic strength of the suspension. Shifting the $\mathrm{pH}$ leads to "more homogeneous" microstructures through diffusion-limited aggregation $(\Delta \mathrm{pH}$-method) while increasing the ionic strength results in "more heterogeneous" microstructures via reaction-rate-limited aggregation ( $\Delta \mathrm{I}$-method). These differences in heterogeneity have been observed using various experimental techniques such as diffusing wave spectroscopy [16], static light transmission [16] or cryogenic scanning electron microscopy [17].

Rheological and uniaxial compression experiments on coagulated colloidal particle structures obtained by direct coagulation casting have revealed that those with a more heterogeneous microstructure have significantly higher elastic moduli than their more homogeneous counterparts [18-20]. The rheological properties, which are the subject of the computational part of this study, were investigated experimentally using a Bohlin rheometer (Model CS-50, Bohlin Instruments, Sweden) equipped with a measuring tool of plate/plate geometry (rough surface, $25 \mathrm{~mm}$ plate diameter). Oscillatory measurements were performed at a fixed frequency of $1 \mathrm{~Hz}$ with increasing strain amplitude. Figure 1 summarizes the measured plateau storage moduli $G_{p}^{\prime}$, i.e. the elastic shear modulus in the linear regime (small deformations), of alumina particle suspensions (average particle diameter $d_{0}=0.4 \mu \mathrm{m}$ ) destabilized by the $\Delta \mathrm{pH}$ - and the $\Delta \mathrm{I}$-method, respectively, as a function of the volume fraction. For this system, approximately four times higher elastic properties are measured for heterogeneous than for homogenous microstructures at corresponding volume fractions [19,21].

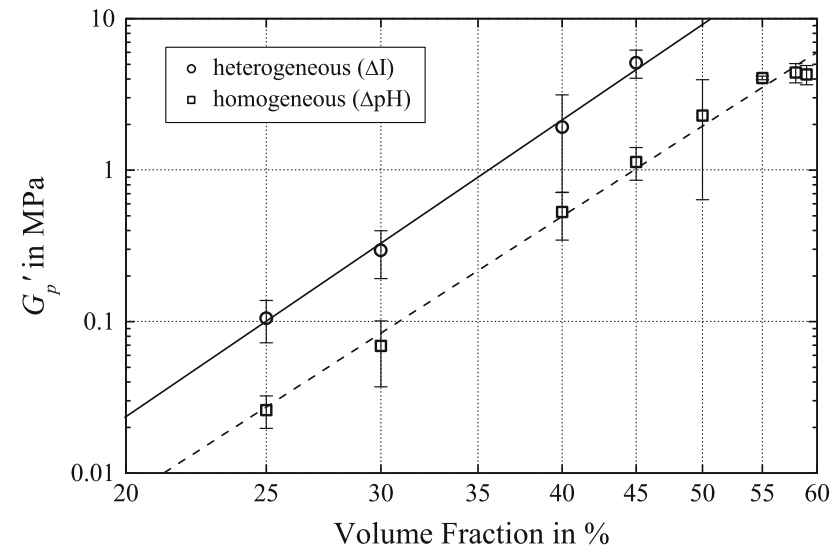

Fig. 1 Experimental plateau storage modulus $G_{p}^{\prime}$ of alumina particle suspensions (average particle diameter $d_{0}=0.4 \mu \mathrm{m}$ ) formed by the $\Delta \mathrm{pH}$ - and the $\Delta \mathrm{I}$-method of the direct coagulation casting process in dependence of the volume fraction [19]

A variation of the $\Delta \mathrm{pH}$-method allowing for a controlled introduction of heterogeneities is the use of alkali-swellable polymer particles. Small amounts of these particles, $80 \mathrm{~nm}$ in diameter, are admixed to the suspended powder particles under acidic conditions. The polymer particles swell upon changing $\mathrm{pH}$ during the internal gelling reaction of the direct coagulation casting process and enfold to $0.7 \mu \mathrm{m}$ in diameter, thereby rearranging the powder particles and thus producing more heterogeneous microstructures. These more heterogeneous samples exhibit much higher mechanical properties in comparison to samples without polymer particles. In particular, they present comparably high mechanical properties as samples with heterogeneous microstructures produced by the $\Delta \mathrm{I}$-method $[22,23]$.

In summary, strong evidence is given that the differences in macroscopic mechanical properties of coagulated particle suspensions are controlled by the differences in heterogeneity. A yet unanswered question is how these microstructural differences on the length scale of a few particle diameters can have such a dramatic influence on the macroscopic mechanical properties.

The aim of this study is to perform a systematic computational analysis of the elastic shear properties of particle packings at constant volume fraction of $\Phi=0.4$ and to correlate these properties with the structures' $\mathrm{DOH}$.

\section{Materials and methods}

In a first part of this section, the discrete element method, which is the simulation method used throughout this study, is introduced. Second, the methods, by which the two sets of initial microstructures are obtained are briefly presented. Then, the method and fit function that is used in order to quantify the structures' DOH is explained and finally, the simulation setup is presented. 


\subsection{Discrete element method}

The discrete element method [24] is a numerical method that allows simulating the motion of large numbers of interacting particles. The simulations presented in this paper are performed using the particle flow code in three dimensions $\mathrm{PFC}^{3 \mathrm{D}}$ [25], which is an implementation of the discrete element method using spherical particles. One calculation step consists in the determination of the total force on each particle and the numerical integration of the equations of motion using a central difference scheme. The force calculation and the determination of the next positions and velocities are then repeated until the end of the simulation.

Our model comprises a linear elastic contact law between particles, particle bonding and damping. The linear elastic contact law relates the contact forces acting on two particles in contact linearly to the relative displacement between the particles. In PFC ${ }^{3 D}$ a soft-contact approach is used, wherein the rigid particles are allowed to overlap at contact points. The magnitude of the normal contact force $F_{n}$ is given by

$F_{n}=k_{n} U_{n}$,

where $k_{n}$ denotes the normal stiffness and $U_{n}$ is the overlap distance $\left(U_{n}>0\right)$. The shear stiffness $k_{s}$ relates incremental displacements in shear direction $\Delta U_{S}$ to the shear contact force $\Delta F_{S}$ via

$\Delta F_{s}=k_{s} \Delta U_{s}$.

The linear elastic contact law is thus parameterized by the normal and shear stiffnesses.

The so-called contact bonds used in $\mathrm{PFC}^{3 \mathrm{D}}$ essentially extend the linear elastic contact law, Eqs. (1) and (2), to particles that are separated, i.e., to particles without overlap $\left(U_{n}<\right.$ $0)$. These bonds are characterized by a normal and shear strengths, $F_{n}^{B}$ and $F_{s}^{B}$. A bond breaks if either the normal or the shear bond strength is exceeded in normal and shear direction, respectively.

Energy dissipation is simulated via a local, non-viscous damping term added to the equations of motion [26]. This damping force $\mathbf{F}_{\mathbf{d}}$ is characterized by the damping coefficient $\alpha$ and is given componentwise (index $i \in\{1,2,3\}$ ) by

$\mathbf{F}_{\mathbf{d}, i}=-\alpha\left|\mathbf{F}_{i}\right| \operatorname{sign}\left(\mathbf{v}_{i}\right)$,

where $\mathbf{F}$ is the force acting on a particle, $\mathbf{v}$ is the particle's velocity and

$\operatorname{sign}(x)=\left\{\begin{array}{rl}+1, & \text { if } x>0 \\ -1, & \text { if } x<0 \\ 0, & \text { if } x=0\end{array}\right.$.

The damping force is thus proportional to the force acting on the particle and indeed leads to energy-dissipation since $\mathbf{F}_{\mathbf{d}} \cdot \mathbf{v}<0$. In particular, only accelerating motion is
Table 1 Simulation parameters

\begin{tabular}{lll}
\hline Parameter & Symbol & Value \\
\hline Number of particles & $N$ & 8,000 \\
Particle radius & $r_{0}$ & $0.25 \mu \mathrm{m}$ \\
Normal particle stiffness & $k_{n}$ & $50-150 \mathrm{~N} / \mathrm{m}$ \\
Shear particle stiffness & $k_{s}$ & $5-15 \mathrm{~N} / \mathrm{m}$ \\
Normal bond strength & $F_{n}^{B}$ & $10^{-4} \mathrm{~N}$ \\
Shear bond strength & $F_{s}^{B}$ & $10^{-6} \mathrm{~N}$ \\
Damping coefficient & $\alpha$ & 0.9 \\
Friction coefficient & $\mu$ & 0.0 \\
Volume fraction & $\Phi$ & 0.4 \\
Particle density & $\rho$ & $3,690 \mathrm{~kg} / \mathrm{m}^{3}$ \\
\hline
\end{tabular}

damped [25]. An analogous expression to Eq. (3) is used to describe the damping of the particles' rotational motion.

In conclusion, the simulation model is characterized by five microscopic parameters: the stiffness of the particles and the bond strength both for normal and shear direction, and the damping coefficient. These parameters, in the following referred to as microparameters, are compiled in Table 1. In particular, the normal to shear particle stiffness ratio $k_{n} / k_{s}=$ 10 is kept constant and a value of 0.9 is chosen for the damping coefficient, which corresponds to the values used in previous studies [10,27]. In $\mathrm{PFC}^{3 \mathrm{D}}$ the frictional term between two particles is superseded in the presence of a bond. However, it may become active in the case of larger deformation when unbonded particles come into contact. In order to prevent such contacts to result in friction, a friction coefficient $\mu=0$ was used. It has to be mentioned that the present paper does not include a detailed sensitivity study of every simulation parameter. Here, the focus is on the influence of the microstructural differences on the mechanical properties. This influence can a priori also be observed with other choices of parameters as long as a unique set is used for all structures.

\subsection{Initial microstructures}

Two sets of microstructures are used as initial particle configurations for the simulation of the elastic properties. A first set originates from previous Brownian dynamics (BD) simulations $[11,28]$ where the coagulation of electrostatically stabilized colloidal suspensions to stiff particle structures was simulated. The presence and depth of a secondary minimum in the inter-particle potential, described by the Derjaguin-Landau-Verwey-Overbeek theory [29], was shown to account for the variations in the $\mathrm{DOH}$ of the resulting particle structures and is adjusted via the surface potential $\Psi_{0}$. For $\Psi_{0}=0 \mathrm{mV}$, the electrostatic double layer repulsion is zero and the inter-particle potential is only given by the attractive van der Waals potential. For the set of 
simulation parameters used in $[11,28]$, a secondary minimum appears for $\Psi_{0} \geq 12 \mathrm{mV}$ and an energy barrier between the local maximum and the secondary minimum emerges. For $\Psi_{0}=15 \mathrm{mV}$, a repulsive barrier of $5.65 k_{B} T$ is present. The model further contains the frictional Stokes' drag force and a random Brownian force caused by the suspending liquid. In this paper, BD-microstructures generated with $\Psi_{0}=0,12,13,14$ and $15 \mathrm{mV}$ are used, exhibiting an increasing DOH with increasing $\Psi_{0}$ [10].

In particular, the most and least heterogeneous BD-microstructures have been shown to nicely correspond to experimental silica structures using the pair correlation function [17]. These two structures are thus used here to calibrate the microparameters in order for the simulations to reproduce the experimental values given in Fig. 1 (cf. Sect. 3.1).

The second set of initial microstructures is obtained using the void expansion method (VEM) presented in [30]. In contrast to $\mathrm{BD}$, which simulates the physical processes during coagulation according to established laws and methods, VEM is a purely stochastic method inspired by the experimental generation of heterogeneous microstructures using alkali-swellable polymer particles. This method employs socalled void-particles that mimic the polymer particles. The void-particles, having a small initial diameter, are randomly placed into the simulation box containing the structure-particles. The core part of VEM is the repeated increase in the void-particles' diameter. During this procedure, the structure-particles are rearranged and pushed into contact. Finally, the void-particles are deleted. As shown in [27], VEM allows generating microstructures presenting a broad range of $\mathrm{DOH}$, which is controlled by the void- to structure-particle number ratio. In particular, VEM-microstructures cover an approximately $20 \%$ larger range of $\mathrm{DOH}$, slightly shifted to higher values than the BD-microstructures. In this study, the number of void-particles $N_{V}$ ranges between 1,000 and 16,000. The void-particles' normal and shear stiffness used in VEM are $k_{n, V}=10^{2} \mathrm{~N} / \mathrm{m}$ and $k_{s, V}=10^{-2} \mathrm{~N} / \mathrm{m}$, respectively.

All structures are submitted to the same relaxation procedure before undergoing the simulated mechanical testing. Bonds between neighboring particles having a center-to-center separation smaller than $d_{\epsilon}=(1+\epsilon) 2 r_{0}$ with $\epsilon=0.01$ are installed. Then, several calculation steps are performed, where, after each step, the translational and rotational velocities are set to zero. This allows for an efficient reduction of particle overlaps and thus of the internal strain energy in the structures without significant changes in the particle positions. The relaxation process is aborted as soon as the mean unbalanced force in the structure has decreased to a constant value.

The structures investigated in this study have equal volume fraction of $\Phi=0.4$, consist of $N=8,000$ monodispersed spherical particles having a radius $r_{0}=0.25 \mu \mathrm{m}$ and are
Table 2 DOH-parameter $a[10,27]$, coordination number $C$ and simulated plateau storage moduli $G_{p}^{\prime}$ of the various VEM- and BD-microstructures

\begin{tabular}{lllll}
\hline & Structure & $a$ & $C$ & $G_{p}^{\prime}$ in MPa \\
\hline \multirow{4}{*}{ VEM } & $N_{V}=1,000$ & 0.594 & 4.68 & $1.73 \pm 0.383$ \\
& $N_{V}=2,000$ & 0.521 & 4.71 & $1.83 \pm 0.136$ \\
& $N_{V}=4,000$ & 0.467 & 4.69 & $1.62 \pm 0.190$ \\
& $N_{V}=8,000$ & 0.418 & 4.69 & $1.32 \pm 0.237$ \\
& $N_{V}=13,000$ & 0.399 & 4.69 & $1.32 \pm 0.267$ \\
& $N_{V}=16,000$ & 0.392 & 4.61 & $1.17 \pm 0.231$ \\
\hline & $\Psi_{0}=0 \mathrm{mV}$ & 0.373 & 4.52 & $0.480 \pm 0.049$ \\
$\mathrm{BD}$ & $\Psi_{0}=12 \mathrm{mV}$ & 0.404 & 4.84 & $1.19 \pm 0.141$ \\
& $\Psi_{0}=13 \mathrm{mV}$ & 0.410 & 4.75 & $1.05 \pm 0.124$ \\
& $\Psi_{0}=14 \mathrm{mV}$ & 0.472 & 5.08 & $1.46 \pm 0.120$ \\
& $\Psi_{0}=15 \mathrm{mV}$ & 0.536 & 4.97 & $1.62 \pm 0.213$ \\
\hline
\end{tabular}

contained in a cubic simulation box with periodic boundaries and edge length $L_{\text {box }}$ given by

$L_{\text {box }}=r_{0}\left(\frac{4 N \pi}{3 \Phi}\right)^{1 / 3}$.

The structures used in this study are provided as electronic supplementary material (Online Resources 1-12).

\subsection{Degree of heterogeneity}

In [10], three distinct methods allowing for a quantification of the DOH using scalar measures were introduced. Here, the $\mathrm{DOH}$ is characterized by means of the cumulative pore size distribution $P\left(r_{P}>r\right)$ estimated using the exclusion probability [31]. Equation (6) was shown to nicely fit $P\left(r_{P}>r\right)$ of both the VEM- and the BD-microstructures $[10,27]$.

$P\left(r_{P}>r\right)=1-\operatorname{erf}\left(\frac{r / r_{0}-b}{a \sqrt{2}}\right)$.

The DOH is quantified by the width of the error function measured by parameter $a$. The values of $a$ for the various microstructures are summarized in Table 2. Parameter $b$ represents the location of the maximum of the underlying Gaussian distribution, i.e., the most probable pore to particle radius ratio.

Additionally, the structures' coordination numbers $C$ are given in Table 2 and plotted in Fig. 2 against the DOH-parameter $a$. This figure shows that the $\mathrm{DOH}$ and the coordination number are to a large extent independent.

\subsection{Simulation setup}

The simulation setup is schematically shown in Fig. 3 . The structure (white particles) is placed between periodic 


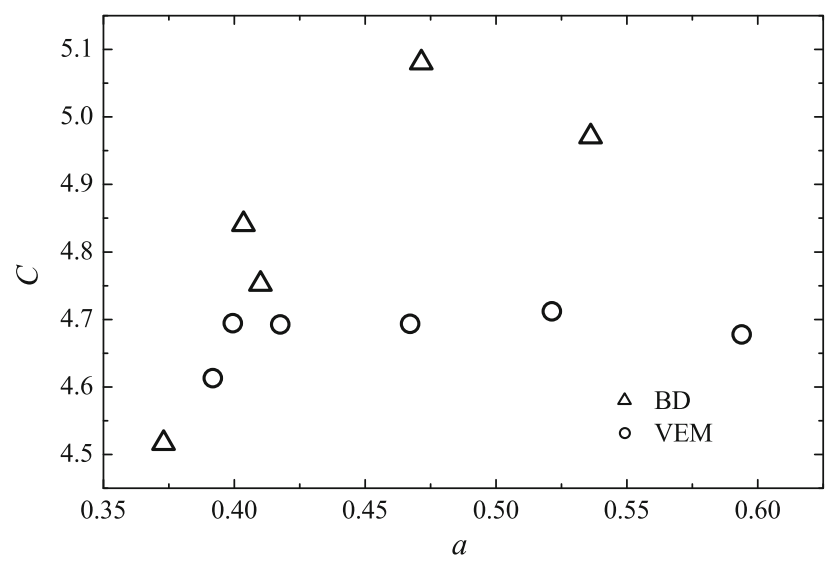

Fig. 2 Degree of heterogeneity as measured by parameter $a$ as a function of the coordination number $C$ for all structures used in this study

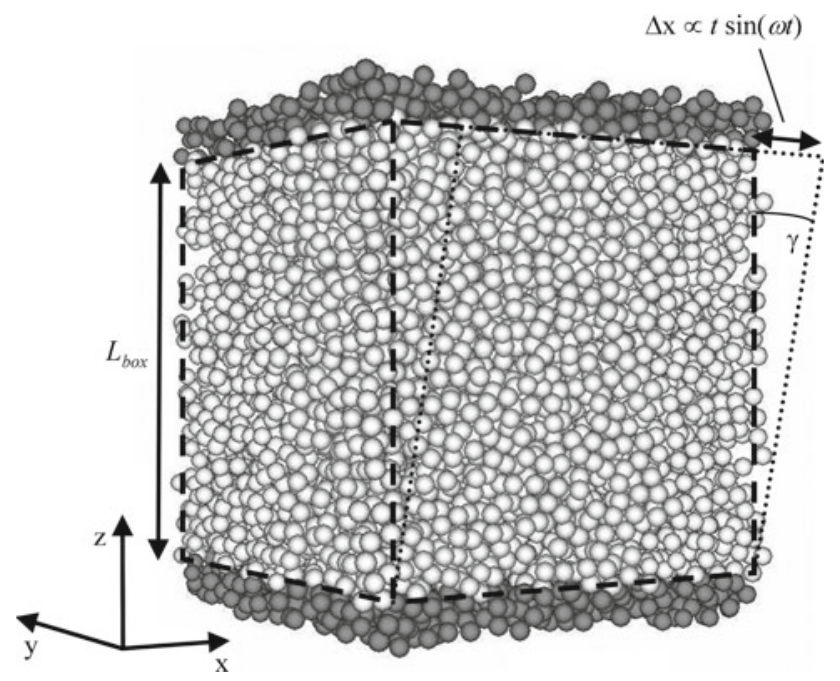

Fig. 3 Rheological simulation setup

boundaries in $\mathrm{x}$ - and $\mathrm{y}$-direction. In z-direction, the boundary conditions are imposed via three additional particle layers (gray), obtained from the periodic repetition of the structure. The lower layers, representing the lower plate in the shear experiment, are immobile whereas the upper layers shear the sample periodically along the $\mathrm{x}$-axis with a fixed frequency of $1 \mathrm{~Hz}$ and constant volume. The shear amplitude is increased after each oscillation. In particular, ten oscillations with a linearly increasing deflection of the upper plate up to a maximum deflection of $0.1 \% L_{\text {box }}$ are performed. Spherical regions, so-called measurement spheres [25], are defined in different heights monitoring the mean stress tensor in their respective region. The absolute value of the complex modulus $G$ is obtained by dividing the peak shear stress value with the actual peak strain of each oscillation. The storage modulus $G^{\prime}$ is then calculated by multiplication with the cosine of the phase angle between excitation and response, which corresponds to the evaluation of $G^{\prime}$ according to [32]. In total, the simulations are performed six times for each structure. The shear plate's normal vector is successively oriented along the $\mathrm{x}-, \mathrm{y}$ - and $\mathrm{z}$-axis and, for each orientation, the structure is sheared along the two remaining axes.

\section{Results and discussion}

\subsection{Calibration of the model}

Typical stress and strain curves are shown in Fig. 4, presenting the shear stress $\sigma_{12}$ (open squares, left scale) and the applied shear deflection $\gamma_{12}$ (full circles, right scale) as a function of the shear oscillation cycle. $\sigma_{12}$ increases linearly with $\gamma_{12}$ and no phase shift between excitation and response is observed (phase angle $<10^{-4} \pi$ ). This confirms that the simulations present a purely elastic behavior and the constant ratio $\sigma_{12} / \gamma_{12}$ is identified with the plateau storage modulus $G_{p}^{\prime}$.

The particle stiffness $k_{n}$ has been calibrated using the most homogeneous and the most heterogeneous BD-microstructures ( $\Psi_{0}=0 \mathrm{mV}$ and $\Psi_{0}=15 \mathrm{mV}$, respectively) using a constant ratio $k_{n} / k_{s}=10$. Its influence on the mechanical properties of these two structures is shown in Fig. 5 (left graph). The experimental values are shown on the right.

The simulated values for $G_{p}^{\prime}$ quantitatively agree with the experimental values within the experimental error for normal stiffness values $k_{n}$ ranging between 50 and $150 \mathrm{~N} / \mathrm{m}$. For both microstructures, a linear dependency is found between $G_{p}^{\prime}$ and $k_{n}$, which is expressed in a constant ratio between the $G_{p}^{\prime}$-values for the heterogeneous and homogeneous microstructure as a function of $k_{n}: G_{p}^{\prime \mathrm{HE}} / G_{p}^{\prime \mathrm{HO}} \approx 3.4$. This is in good agreement with the average experimental $G_{p}^{\prime}$-ratio of 3.6 at $\Phi=0.4$. For all further simulations the particle normal stiffness is fixed to $k_{n}=100 \mathrm{~N} / \mathrm{m}$.

The inter-particle bond strength was strictly speaking not part of the calibration process. In order to focus on the ini-

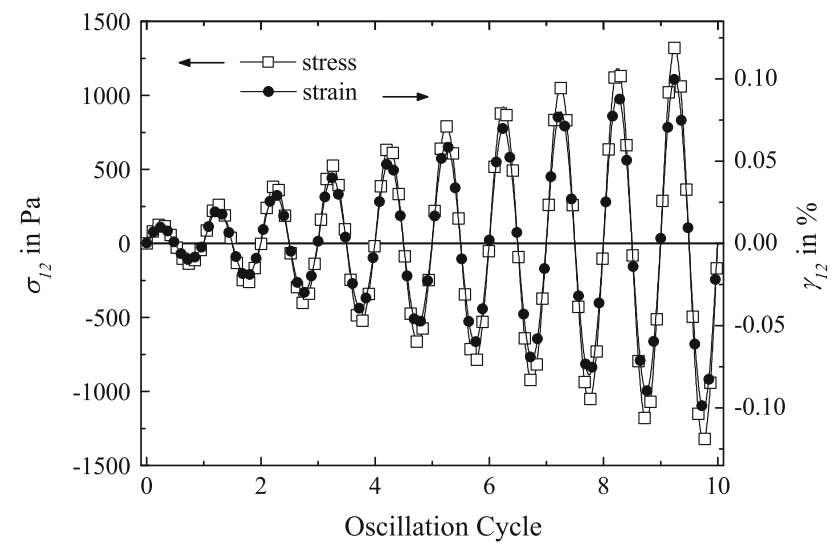

Fig. 4 Stress $\sigma_{12}$ (open squares, left scale) and strain $\gamma_{12}$ (full circles, right scale) as a function of shear oscillation cycle 


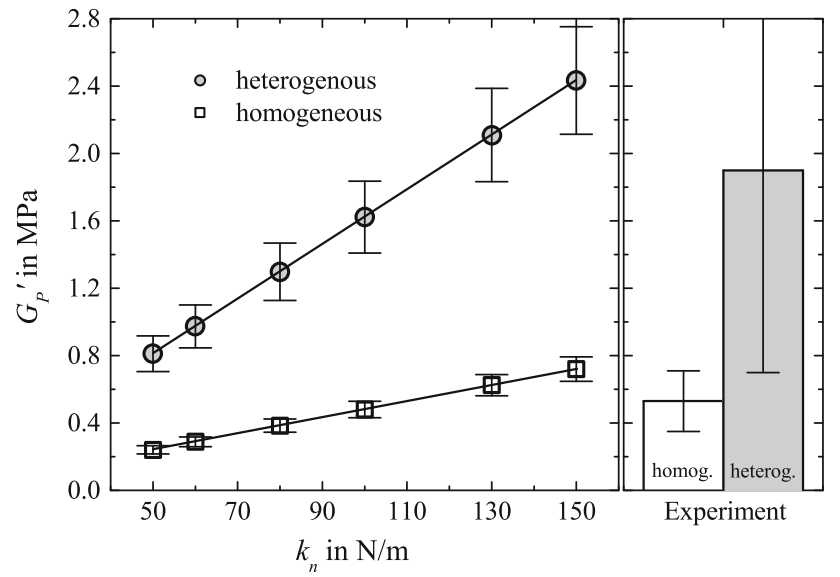

Fig. 5 Simulated plateau storage moduli $G_{p}^{\prime}$ of the most and least heterogeneous BD-microstructure as a function of the particle normal stiffness $k_{n}$. Right: Experimental $G_{p}^{\prime}$-values for alumina particle structures (cf. Fig. 1). $\Phi=0.4$ for all microstructures

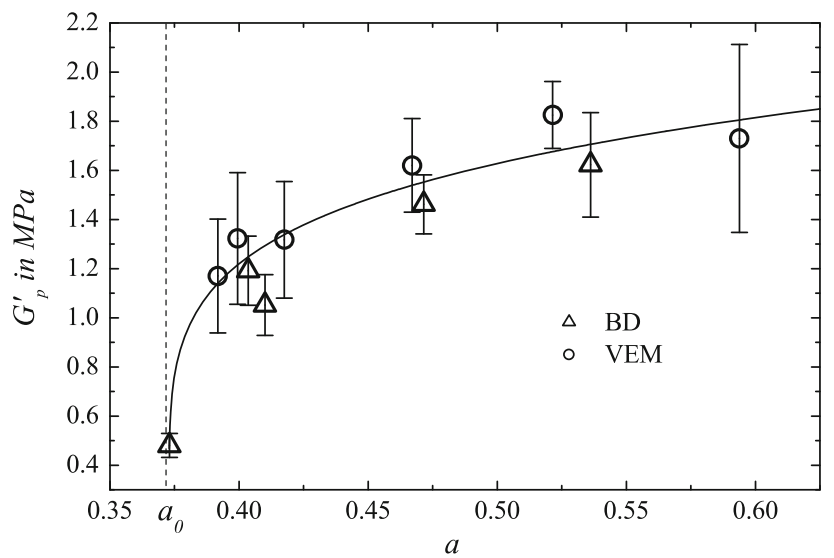

Fig. 6 Simulated plateau storage moduli $G_{p}^{\prime}$ of the various microstructures $\left(\Phi=0.4, r_{0}=0.25 \mu \mathrm{m}\right)$ as a function of the $\mathrm{DOH}$ in terms of parameter $a$ and power law fit (solid line)

tial elastic properties, its value was chosen high enough to prevent any bond breakage for the deformations considered in this study. The chosen normal bond strength $F_{n}^{B}=10^{-4} \mathrm{~N}$, in conjunction with the particle normal stiffness $k_{n}=100 \mathrm{~N} / \mathrm{m}$ allows for a maximum particle separation distance of $10^{-6} \mathrm{~m}$, which corresponds to two particle diameters. Given the maximum shear displacement of $0.001 L_{\text {box }}$, where $L_{\mathrm{box}}$ is of the order of roughly 20 particle diameters, this value is never exceeded.

\subsection{Elastic properties as a function of the $\mathrm{DOH}$}

Using a particle normal stiffness of $100 \mathrm{~N} / \mathrm{m}$, the plateau storage moduli of the various VEM- and BD-microstructure were simulated. The resulting $G_{p}^{\prime}$-values are summarized in Table 2 and are shown in Fig. 6 as a function of the structures' DOH expressed by parameter $a$. Triangles and circles corre- spond to BD- and VEM-microstructures, respectively. Each data point in Fig. 6 corresponds to one structure sheared in all six directions. The error bars are thus reflecting the anisotropy of the structures.

Two conclusions can be drawn from Fig. 6: first, the elastic properties present a clear dependence on the microstructure's heterogeneity such that $G_{p}^{\prime}$ increases for increasing DOH-parameter $a$. Second, the behavior is independent of the structure generation algorithm. Indeed, the elastic moduli of the VEM- and the BD-microstructures agree within the error bars for microstructures with comparable DOH.

The solid line in Fig. 6 represents a power law fit given by $G_{p}^{\prime} \propto\left(a-a_{0}\right)^{\beta}, a \geq a_{0}$,

with $a_{0}=0.373 \pm 0.001$ (shown as dashed line in Fig. 6) and $\beta=0.187 \pm 0.032$. The use of this fit function is inspired by percolation theory where a power law scaling is found for the elastic properties as a function of the volume fraction $[33,34]$. In view of this analogy and based on the simulation data currently available, this fit function suggests a phase transition with $a_{0}$ the critical DOH. Below this value, the elastic properties are zero; above this value, they increase for increasing $a$. A DOH-value $a=0.265$ below $a_{0}$ was found for the stabilized colloidal microstructures with repulsive inter-particle potentials [10]. In the model used in this work, this microstructure would indeed present negligible elastic properties since there would be no bonds between the particles.

It has to be mentioned that percolating structures presenting a DOH below the critical value exist. Such structures, as for example intermediate steps during the simulated coagulation toward the most homogeneous microstructure using $\mathrm{BD}$, are not expected to have zero elastic properties. However, they have an average coordination number significantly below the range considered in this study and are therefore not included. Also, it has to be emphasized that the obtained value for $a_{0}$ does not solely rely on the data point possessing the lowest $a$-value. Indeed, a power law fit without this data point yields $a_{0}^{\prime}=0.360$, which is roughly $4 \%$ below $a_{0}$.

The uncertainty of the value for the critical DOH $a_{0}$ roots in fitting the power law (Eq. 7) to the simulated plateau storage moduli of the samples studied here using error propagation. The limits for $a_{0}$ do therefore not represent an uncertainty obtained by analyzing a statistically significant control sample. A more extensive study, involving a larger number of microstructures possessing a $\mathrm{DOH}$ in the immediate vicinity of $a_{0}$, i.e. between 0.35 and 0.4 , would be required in order to determine $a_{0}$ more accurately.

\section{Summary and conclusions}

In this paper, the relation between the microstructure and the elastic properties of packings of spherical particles 
was investigated computationally using the discrete element method. Two distinct sets of initial microstructures were subjected to strain-controlled oscillatory shear simulations. The first set originated from Brownian dynamics simulations $[11,28]$ where the physical processes during coagulation were simulated. The second set was obtained using the void expansion method [30]. In contrast to Brownian dynamics simulations, the latter is a purely stochastic method. Both sets cover a broad range of degrees of heterogeneity, which, in this work, was characterized using the width of the pore size distribution.

The simulations of the mechanical properties have been performed using a discrete element model with five microparameters: the particle stiffness and the bond strength in normal and shear direction, respectively and damping. The inter-particle normal and shear bond strengths were set to a high value in order to prevent any bond breaking for the deformations applied during the shear simulations. This allowed focussing on the initial, purely elastic behavior found in experiment [20]. The particle stiffness was calibrated using the two Brownian dynamics microstructures presenting the highest and lowest DOH, which correspond to experimental silica structures [17].

A quantitative agreement with experiment was achieved. For particle normal stiffnesses ranging between 50 and $150 \mathrm{~N} / \mathrm{m}$, the absolute values of the experimental elastic moduli are reproduced, as shown in Fig. 5. In particular, the ratio between the elastic moduli of the heterogeneous and the homogeneous microstructures is in good agreement with the experimental value. This latter result is particularly remarkable, since the $G_{p}^{\prime}$-ratio has not been subject of any calibration but reflects the influence of the microstructures and emphasizes the strong influence of the DOH independently of the interaction potential. Indeed, the model used in this study constitutes an important simplification with respect to theoretical models such as the Derjaguin-LandauVerwey-Overbeek theory [29] or the Johnson-Kendall-Roberts theory [35]. The former gives the inter-particle potential as the sum of the van der Waals attraction and the electrostatic repulsion and the latter describes the adhesive force between particles.

Using the calibrated model, the elastic properties of all microstructures generated using the void expansion method and Brownian dynamics were simulated. This investigation has revealed a correlation between the elastic properties and the structures' DOH. It has also shown that the elastic properties are independent of the structure generation algorithm. Indeed, for structures with comparable degrees of heterogeneity, comparable plateau storage moduli were found. A power law fit was shown to well reproduce the relation between the plateau storage modulus and the $\mathrm{DOH}$ above a critical value, which indicates a phase transition between a phase with finite and a phase with zero elastic properties. This result is in nice analogy to earlier findings [33,34], where the elastic properties were shown to exhibit a power law dependence as a function of the volume fraction. This study thus suggests an extension to percolation theory, where usually the volume fraction constitutes the continuous variable presenting a critical value. Here, the DOH, possessing the same critical behavior as the volume fraction, has been identified as an additional variable.

The results presented in this paper show that the degree of heterogeneity is particularly useful in order to quantify and characterize the heterogeneity of particle packings since it allows for an unprecedented theoretical description of their elastic properties. However, it does not directly lead to a better understanding of the mechanisms, by which the elastic properties increase for increasing heterogeneity. Such an understanding must, in some way, include an intermediate step, such as an analysis of the distribution of particle contacts using the fabric tensor, for example [36], or of load-bearing substructures, as discussed in [12].

Acknowledgements The authors would like to thank Hans J. Herrmann and Tomaso Aste for their valuable help throughout this project.

\section{References}

1. Fakhimi, A., Villegas, T.: Rock Mech. Rock Eng. 40, 193 (2007)

2. Rognon, P.G., Roux, J.N., Wolf, D., Naaïm, M., Chevoir, F.: Europhys. Lett. 74, 644 (2006)

3. Ottino, J.M., Khakhar, D.V.: Annu. Rev. Fluid Mech. 32, 55 (2000)

4. Zaccone, A., Lattuada, M., Wu, H., Morbidelli, M.: J. Chem. Phys 127, 174512 (2007)

5. Gardiner, B.S., Tordesillas, A.: Powder Technol. 161, 110 (2006)

6. Silbert, L.E., Ertaş, D., Grest, G.S., Halsey, T.C., Levine, D.: Phys. Rev. E 65, 031304 (2002)

7. Martin, C.L., Bordia, R.K.: Phys. Rev. E 77, 031307 (2008)

8. Cates, M.E., Wittmer, J.P., Bouchaud, J.P., Claudin, P.: CHAOS 9, 511 (1999)

9. Atman, A.P.F., Brunet, P., Geng, J., Reydellet, G., Combe, G., Claudin, P., Behringer, R.P., Clément, E.: J. Phys. Condens. Matter 17, S2391 (2005)

10. Schenker, I., Filser, F.T., Aste, T., Herrmann, H.J., Gauckler, L.J.: Phys. Rev. E 80, 021302 (2009)

11. Hütter, M.: J. Colloid Interface Sci. 231, 337 (2000)

12. Schenker, I., Filser, F.T., Aste, T., Gauckler, L.J.: J. Eur. Ceram. Soc. 28, 1443 (2008)

13. Hütter, M.: Phys. Rev. E. 68, 031404 (2003)

14. Gauckler, L.J., Graule, T., Baader, F.: Mater. Chem. Phys. 61, 78 (1999)

15. Tervoort, E., Tervoort, T.A., Gauckler, L.J.: J. Am. Ceram. Soc. 87, 1530 (2004)

16. Wyss, H.M., Romer, S., Scheffold, F., Schurtenberger, P., Gauckler, L.J.: J. Colloid Interface Sci. 241, 89 (2001)

17. Wyss, H.M., Hütter, M., Müller, M., Meier, L.P., Gauckler, L.J.: J. Colloid Interface Sci. 248, 340 (2002)

18. Balzer, B., Hruschka, M.K.M., Gauckler, L.J.: J. Colloid Interface Sci. 216, 379 (1999)

19. Wyss, H.M., Deliormanli, A.M., Tervoort, E., Gauckler, L.J.: AIChE J. 51, 134 (2005)

20. Wyss, H.M., Tervoort, E., Meier, L.P., Müller, M., Gauckler, L.J.: J. Colloid Interface Sci. 273, 455 (2004) 
21. Balzer, B., Hruschka, M.K.M., Gauckler, L.J.: J. Am. Ceram. 84, 1733 (1999)

22. Hesselbarth, D., Tervoort, E., Urban, C., Gauckler, L.J.: J. Am. Ceram. Soc. 84, 1689 (2001)

23. Hesselbarth, D.: Quellfähige Polymerbinder in AluminiumoxidSuspensionen (Ph.D. thesis no. 13404, ETH Zurich, Switzerland, 2000) http://e-collection.ethbib.ethz.ch

24. Cundall, P.A., Strack, O.D.L.: Géotechnique. 29, 47 (1979)

25. PFC $^{3 D}$ User's manual: (Itasca Consulting Group, Inc., Minneapolis, Minnesota, USA) (1995)

26. Brown, E.T.: Analytical and computational methods in engineering rock mechanics. Allen \& Unwin, London (1987)

27. Schenker, I., Filser, F.T., Gauckler, L.J.: Granul. Matter. 12, 437 (2010)

28. Hütter, M.: Brownian dynamics simulation of stable and of coagulating colloids in Aqueous Suspension (Ph.D. thesis no. 13107, ETH Zurich, Switzerland, 1999) http://e-collection.ethbib.ethz.ch
29. Russel, W.B., Saville, D.A., Schowalter, W.R.: Colloidal dispersions. Cambridge University Press, New York (1989)

30. Schenker, I., Filser, F.T., Herrmann, H.J., Gauckler, L.J.: Granul. Matter. 11, 201 (2009)

31. Torquato, S., Lu, B., Rubinstein, J.: Phys. Rev. A. 41, 2059 (1990)

32. Yanez, J.A., Shikata, T., Lange, F.F., Pearson, D.S.: J. Am. Ceram. Soc. 79, 2917 (1996)

33. Benguigui, L.: Phys. Rev. Lett. 53, 2028 (1984)

34. Bergman, D.J.: Phys. Rev. E. 65, 026124 (2002)

35. Johnson, K.L., Kendall, K., Roberts, A.D.: Proc. R. Soc. Lond. A. 324, 301 (1971)

36. Madadi, M., Tsoungui, O., Lätzel, M., Luding, S.: Int. J. Sol. Struct. 41, 2563 (2004) 